\title{
A Novel Correlation of Preoperative Gd-EOB-DTPA- contrast-enhanced MRI with FGFR4 Expression and Its Value in Targeted Therapy for Hepatocellular Carcinoma
}

\section{Zhiqing Mo}

First Affiliated Hospital of GuangXi Medical University

Liling Long ( $\sim$ Cjr.longliling@vip.163.com )

First Affiliated Hospital of GuangXi Medical University

Hao Ding

First Affiliated Hospital of GuangXi Medical University

Xiaojiao Zhou

First Affiliated Hospital of GuangXi Medical University

\section{Research Article}

Keywords: Hepatocellular carcinoma, magnetic resonance imaging (MRI), Gd-EOB-DTPA-enhanced MRI, FGFR4, targeted cancer therapy

Posted Date: April 21st, 2021

DOI: https://doi.org/10.21203/rs.3.rs-422223/v1

License: (9) This work is licensed under a Creative Commons Attribution 4.0 International License.

Read Full License 
Gd-EOB-DTPA-contrast-enhanced MRI with FGFR4 expression and its value in targeted therapy for hepatocellular carcinoma

Authors: 1Zhiqing Mo, MM,e-mail:1013193428@qq.com Tel: 18078353455

*Liling Long, MD, e-mail:Cjr.longliling@vip.163.com Tel:13807712604

2Hao Ding, MM ,e-mail:410300279@qq.com Tel:18172660915

${ }^{3}$ Xiaojiao Zhou, MM ,e-mail:15082768538@163.com Tel:15082768538

Institutions: Department of Radiology, the First Affiliated Hospital of Guangxi Medical University, Nanning, Guangxi 530021, China

*Corresponding author: Liling Long, MD

Department of Radiology

The First Affiliated Hospital of Guangxi Medical University

Nanning, Guangxi 530021, China

Tel: +86-13807712604 
E-mail:Cjr.longliling@vip.163.com

\begin{abstract}
Purpose: To assess the relationship between preoperative gadolinium ethoxy-benzyl diethylenetriaminepentaacetic acid (Gd-EOB-DTPA)-enhanced magnetic resonance imaging (MRI) features and fibroblast growth factor receptor 4 (FGFR4) gene expression in hepatocellular carcinoma (HCC).

Materials and methods: Fifty-nine HCC patients (54 males, 5 females) who underwent preoperative enhanced MRI were retrospectively enrolled in this study. Quantitative and qualitative features of Gd-EOB-DTPA-enhanced MRI were analyzed in these pathologically confirmed HCC patients. Immunohistochemistry (IHC) and reverse transcription-polymerase chain reaction (RT-PCR) were performed to determine the mRNA and protein levels of FGFR4 in HCC. The relationship between these image features and the level of FGFR4 gene expression in HCC was evaluated by correlation analysis.
\end{abstract}

Results: The FGFR4 mRNA and protein expression has significant correlation with the change of signal intensity in the phase of hepatobiliary, IHC analysis revealed significant correlation between the protein expression of FGFR4 and the qualitative enhanced MRI feature, mainly the manifestation of the intratumoral vessels at the arterial phase. Furthermore, the presence of intratumoral vessels $(P=0.034, O R=4.71)$ and heterogeneous 
signal performance in the hepatobiliary phase $(P=0.008, O R=4.2)$ were identified as independent indicators for high FGFR4 expression in HCC.

Conclusions: The findings demonstrate novel correlation between enhanced MRI features and FGFR4 gene expression, suggesting the heterogeneous signal intensity at the phase of hepatobiliary and the present of intratumoral vessels in the arterial phase as indicators for high FGFR4 expression in HCC. Our study may have clinical implication that enhanced MRI holds promise as useful modality in treatment selection of targeted therapies to HCC patients.

Keywords: Hepatocellular carcinoma, magnetic resonance imaging (MRI), Gd-EOB-DTPA-enhanced MRI, FGFR4, targeted cancer therapy

\section{Key Points}

- Heterogeneous signal intensity at the phase of hepatobiliary and the presence of Intratumoral vessels in the arterial phase on enhanced MRI were independent indicators for high FGFR4 expression in HCC.

- Enhanced MRI is potential modality in treatment selection and efficacy evaluation of targeted therapies for HCC patients. 


\section{Introduction}

HCC is the fifth most common cancer worldwide and the fourth leading cause of cancer-related death, mainly attributed to relatively high incidence of hepatitis B virus (HBV)-induced HCC and delayed diagnosis of $\mathrm{HCC}$ at late stages[1, 2]. Among the treatments for advanced HCC, targeted cancer therapy, which specifically interferes with target molecules to block the tumor growth and spread, has emerged as a new generation of treatment.

Fibroblast growth factor receptor 4 (FGFR4), a member of the FGFR (1-4) family, is a kind of tyrosine kinase receptor with the ligand fibrocyte growth factor 19(FGF19) that mediates cellular effects by stimulating autophosphorylation in reverse transcription and downstream MAP kinase 、 Akt signaling pathways[3]. It has been shown that the interactivity between FGF19 and its specific receptor FGFR4 contributes to the tumorigenesis of liver cancer[4]. Recently, several FGFR4 inhibitors, with different binding patterns and selectivity, have been developed and assessed in clinical trials for the treatment to HCC with abnormally high FGFR4 expression[5]. For instance, Lenvatinib is a potent tyrosine receptor kinase inhibitor targeting the vascular endothelial growth factor receptor (VEGFR1-3), fibroblast growth factor receptor1-4 (FGFR1-4), KIT and RET. Compared with sorafenib, Lenvatinib is the second and most commonly used first-line targeted drug for HCC approved by the American Food and Drug 
Administration (FDA)[6]. Lenvatinib is not inferior to sorafenib for improving tumor-free to progression and overall survival rate, is indeed superior to sorafenib and is more cost effective[7]. Although targeted drug therapy can improve the outcome of a proportion of patients with advanced $\mathrm{HCC}$, it has been noted that not all patients benefit from Lenvatinib. Differences in the clinical prognosis in patients receiving the targeted cancer therapy may be due to the heterogeneity of target genes for $\mathrm{HCC}[8]$. It has been noted that FGFR4 gene express level is an independent predictor for reaction to Lenvatinib treatment and is related with longer progression-free survival and good objective response rate[9]. The study showed that FGFR4-positive tissues were related with longer progression-free survival $(2.5 \mathrm{vs} 5.5$ months, $\mathrm{P}=0.01)$ and better objective response rates $(31 \%$ vs $81 \%, P=0.006)$. Therefore, these findings highlight importance of detecting the expression of target genes before targeted cancer therapy to achieve high therapeutic efficacy at low cost.

Currently, preoperative genetic testing for $\mathrm{HCC}$ is mainly performed by biopsy. However, because of the genetic heterogeneity in cancer tissues, biopsy specimen may not reflect the overall tumor characteristics. In addition, the procedures are invasive, leading to increase in the risk of procedure-associated complications (e.g. hemorrhage, tumor spread). Notably, preoperative enhanced MRI allows examination of the overall 
tumor tissues, for which their corresponding characteristics can be better analyzed[10]. In fact, some MRI features of cancer tissues were significantly correlated with the mutated B-RAF and RAF-1 gene expression[11]. Hectors et al[12]proposed that the association of 28 imaging signs could predict the expression of $74 \%$ of 6732 genes, and many global gene expression profiles for $\mathrm{HCC}$ were related to their imaging characteristics. A previous study has revealed a correlation between contrast-enhanced $\mathrm{MRI}$ features on T1-weighted/diffusion weighted images, and programed death-1 (PD -1)/programed death-ligand 1 (PD-L1) and cytotoxic T-lymphocyte-associated antigen 4 (CTLA4), the targets for immunotherapeutic cancer treatments[13]. Until now, it remains unclear whether and which MRI features could reflect the FGFR4 gene expression in HCC.

In this research, we sought to evaluate the connection between preoperative Gd-EOB-DTPA-enhanced MRI image features and FGFR4 gene expression in HCC patients. The finding obtained through conducting this study may provide scientific evidence for the clinical use of Gd-EOB-DTPA-enhanced MRI to select targeted therapies for HCC patients. 


\section{Materials and methods}

\section{Study patients}

In this study, we retrospectively collected and analyzed 59 patients with HCC who were diagnosed and treated in the First Affiliated Hospital of Guangxi Medical University (Guangxi, China) spanning from April 2019 to December 2020. Inclusion criteria were as follows :(1) HCC was pathologically diagnosed and confirmed after surgery; (2) Pre-operative Gd-EOB-DTPA enhanced MRI examination was performed;(3) No distant metastasis;(4) Surgical treatment was conducted within 2 weeks after the enhanced MRI examination;(5) HCC was not treated before MR examination and surgical resection. The patients with the following conditions were excluded in this study: (1) No pre-operative Gd-EOB-DTPA enhanced MRI scan;(2) medical history with other malignant tumors;(3) incomplete pathological and/or clinical information; (4) poor quality of MRI images.

\section{MRI acquisition and image analysis}

All patients underwent Gd-EOB-DTPA (Bayer Healthcare Co., Ltd., Germany)-enhanced MRI examination on the 3.0 T MR scanner (German Siemens) 2 weeks before surgery. Gd-EOB-DTPA (dosage, $0.025 \mathrm{mmol} / \mathrm{kg}$ ) was injected at a flow rate of $2 \mathrm{ml} / \mathrm{s}$ through the anterior cubital vein. Normal saline $(20 \mathrm{ml})$ was then injected at the flow rate of $2 \mathrm{ml} / \mathrm{s}$ for flushing. The following main sequences and parameters were used: T1-weighted image (T1WI), T2-weighted image (T2WI), 
diffusion-weighted image (DWI, $\left.b=50,800 \mathrm{~s} / \mathrm{mm}^{2}\right)$, T1 mapping. MRI scans were performed in the arterial phases, portal vein phases, equilibrium phases and hepatobiliary phases at $14-20 \mathrm{~s}, 40-50 \mathrm{~s}, 90-120 \mathrm{~s}$, and 20 min after contrast agent injection, respectively.

Two experienced radiologists analyzed MRI images blind to pathological findings. Qualitative MRI features, including tumor thrombosis, T2WI and hepatobiliary phase signal intensity, tumor capsule status, tumor margin, Intratumoral vessels, Intratumoral hemorrhage and intratumoral fat were determined by two radiologists. Qualitative MRI features were characterized (Fig. 1):(1) Tumor thrombosis: portal vein or hepatic vein filling defect, obvious enhancement at the phase of arterial, obvious flushing at the phase of portal vein;(2) Signal intensity: T2WI transverse signal intensity uniformity was evaluated, and hepatobiliary phase signals were evaluated by uniform low signal, heterogeneous of high and low signal; (3) tumor capsule: The high signal thin margin of the tumor margin in the equilibrium phase is considered as the tumor capsule. Tumors are defined as either capsule or not capsule according to the capsule sign. The capsule neoplasms were further divided into completely capsule and incomplete capsule groups; (4) tumor margin: Depending on the edge of the hepatobiliary phase, there are four types of tumor margin : smooth isolated type, protruding type, irregular infiltrating and multiple nodular fusion type;(5) intratumoral vessels: in the arterial phase, blood vessels can be seen within the tumor. Quantitative characteristics included tumor size in diameter, apparent diffusion coefficient (ADC) value, T1 percentage decline (T1D\%). Based on coronal and axial images, the maximum tumor diameter was measured at the phase of hepatobiliary. ADC values, non-enhanced $\mathrm{T} 1$ relaxation time $(\mathrm{T} 1 \mathrm{~N})$ and hepatobiliary phases (T1E) were all at the solid component level of HCC to avoid necrosis, blood vessels and artifacts. T1 relaxation reduction rate $\mathrm{T} 1 \mathrm{D} \%$ was calculated in the hepatobiliary phase: $\mathrm{T} 1 \mathrm{D} \%=(\mathrm{T} 1 \mathrm{~N}-\mathrm{T} 1 \mathrm{E}) / \mathrm{T} 1 \mathrm{~N}[11]$. 


\section{Immunohistochemical analysis}

Immunohistochemistry (IHC) was used to detect the protein expression level of FGFR4 in HCC specimens. The primary antibody is mouse monoclonal antibody (5B5-ab44971 /FGFR4 antibody) used for immunohistochemical staining of all nodular specimens with FGFR4, The second antibody is goat anti-mouse/rabbit IgG polymer (SP-9000). Intratumoral specimens were taken from 1-5 sites of the primary lesion to avoid necrosis and bleeding. All specimens were stained with FGFR4 immunohistochemistry and the FGFR4 positive staining was defined as brown I yellow staining of the cell cytoplasm and membrane. In this study, after hematoxylin reverse staining, the percentage of FGFR4 positive cells was quantified microscopically by two independent examinators. The IHC staining of the FGFR4 protein expression was categorized: Grade1with staining in $<10 \%$, Grade 2 with staining in $10 \%-50 \%$, and Grade3 with staining in $\geq 50 \%$ for tumor cells [9](Fig. 2).

\section{RNA extraction and RT-PCR}

Total RNA was extracted from fresh collected specimens using Trizol reagent (Invitrogen) in strict accordance with standard operating instructions. Reverse transcription -polymerase chain reaction (RT-PCR) was performed to amplify target RNA, during which the following primers were used: FGFR4: forward ATTGCCAGCTTCCTACCTGAG, reverse GGGGTAACTGTGCCTATTCG. The internal reference primer is GADPH. SYBR primers and miRNA reverse transcription polymerase chain reaction kits are used for polymerase chain reaction amplification. Then Ct value of the sample was obtained according to the analytical dissolution curve and the real-time amplification curve. The FGFR4 gene expression in the HCC tissues was found to be relative to that of the adjacent tissues $\left(\triangle \mathrm{Ct}\right.$ caliv), as calculated using the following formula: $2^{-\triangle \Delta \mathrm{Ct}}$ Where, $\triangle \triangle \mathrm{Ct}=$ 
$\triangle \mathrm{Ct}$ ca- $\triangle \mathrm{Ct}$ caliv,$\triangle \mathrm{Ct}$ ca $=\mathrm{Ct}$ ca-Ct GADPH,$\triangle \mathrm{Ct}$ caliv $=\triangle \mathrm{Ct}$ caliv- $\mathrm{Ct} \mathrm{GADPH}[14]$. The relationship between FGFR4 gene expression and MRI qualitative and quantitative features was analyzed.

\section{Statistical analysis}

IBMSPSS23.0 (Chicago, IL, USA) was used for all statistical analyses. Nonparametric tests were performed to assess the differences in the qualitative features of different MRI groups. The difference between the MRI features of multiple groups was determined by the Kruskal-Wallis $\mathrm{H}$ test. Spearman rank correlation analysis was performed to assess the relationship between quantitative MRI features and gene expression. The variables with statistical differences in univariate analysis were input into the logistic regression model. The screening adopts positive (conditional) method, the inclusion standard is 0.05 , the exclusion standard is 0.1 . P $<0.05$ was considered statistically significant.

\section{Results}

\section{FGFR4 gene expression in HCC}


FGFR4 gene expression was examined in HCC tissues. Immunohistochemical staining for FGFR4 was performed in 59 HCC tissues, indicating its protein expression $<10 \%, 10 \%-50 \%$, and $\geq 50 \%$ in 26 , 17 , and 16 cases, respectively. RT-PCR analysis of FGFR4 mRNA expression levels was conducted in 48 HCC tissues, of which 16 were positive while 32 were negative for FGFR4 gene expression.

\section{Qualitative evaluation of Gd-EOB-DTPA enhanced MRI features in HCC}

Among 59 HCC patients, tumor thrombosis occurred in 10 cases $(16.9 \%)$, while the majority of the patients $(49,83.1 \%)$ had no thrombosis. The qualitative imaging signs of Gd-EOB-DTPA-enhanced MRI (e.g. signal intensity, enhancement, patterns of malignant nodules) were characterized in $59 \mathrm{HCC}$ patients and the results were summarized in Table 1. An inhomogeneous signal intensity was identified in 55 cases, accounting for as high as $92.7 \%$, while homogenous signal intensity was detected in 4 cases $(7.3 \%)$. In addition, a large proportion of HCC tissues $(43 / 59,72.9 \%)$ showed hypo-intensity in the hepatobiliary phase, and the remaining 16 cases $(27.1 \%)$ exhibited a heterogeneous signal intensity of both hypoand hyper-intensity in the hepatobiliary phase. There were 23 cases with complete capsule (47.5\%), 13 cases with incomplete capsule $(22 \%)$, and 18 cases without capsule $(30.5 \%)$. Patterns of the malignant nodules showed smooth single the nodules in 23 cases (39\%), prominent nodules in 
24 cases $(40.7 \%)$, multiple nodules fusion in 11 cases $(18.6 \%)$, and infiltrating type in 1 case $(1.7 \%)$. Characterization of tumor vessels revealed 22 cases $(37.3 \%)$ with tumor vessels, 37 cases $(62.7 \%)$ with no vessels, of which 23 cases (39\%) with hemorrhage, and 36 cases $(61 \%)$ without hemorrhage. Fat-containing malignancies were identified in 5 cases $(8.5 \%)$ with 54 cases $(91.5 \%)$ in the absence of intratumoral fat.

\section{Quantitative evaluation of Gd-EOB-DTPA-enhanced MRI features in HCC}

The quantitative enhanced MRI features [e.g. tumor size, apparent diffusion coefficient $(A D C)$ on diffusion-weighted image (DWI), value of $\mathrm{T} 1$ mapping for assessment $\mathrm{T} 1$ relaxation reduction in $\mathrm{HCC}$. The size of $59 \mathrm{HCC}$ lesions ranged from 24.00 to $159.00 \mathrm{~mm}$ in diameter (mean, $66.4 \mathrm{~mm}$; SD, $37.10 \mathrm{~mm}$ ). DWI $\left(b=800 \mathrm{~s} / \mathrm{mm}^{2}\right.$ ) was performed to measure ADC value in $59 \mathrm{HCC}$ cases. The range of ADC was $(0.62-1.96) \times 103 \mathrm{~mm}^{2} / \mathrm{s}$, with the average value of $(0.96 \pm 0.21) \times 103 \mathrm{~mm}^{2} / \mathrm{s}$. There are 22 cases were performed T1 mapping to measure $\mathrm{T} 1$ relaxation reduction in $\mathrm{HCC}$. T1D\% ranged from 0.21 to 0.64 with an average of 0.42 .

\section{Correlation analysis of FGFR4 gene expression and Gd-EOB-DTPA-} enhanced MRI features in HCC

We examined the relationship between FGFR4 gene expression and Gd-EOB-DTPA-enhanced MRI features in HCC. Univariate analysis indicated 
that the protein expression level of FGFR4 was statistically significant in the presence or absence of Intratumoral vessels in the arterial phase $(P=0.041)$, and FGFR4 mRNA expression was significantly different between the hepatobiliary hypointense group and heterogeneous of high and low signal group $(P=0.005)$. Other $M R$ qualitative characteristics were not statistically different between the groups (Table 2 and Fig. 3). There was no significant correlation between FGFR4 expression level and MRI quantitative characteristics $(P>0.05)$ (Table 3$)$.

Multivariate logistic regression analysis revealed that the presence of Intratumoral vascular as an independent factor for FGFR4 protein expression $(P=0.034)$. The protein expression level of FGFR4 in the grade 2 group, the expression level of the presence group was higher than that of the non-presence group $(P=0.026, O R=4.71,95 \% \mathrm{Cl}: 1.209-18.518)$. The expression level of FGFR4 in the grade3 group, the presence group was higher than in the absence group $(P=0.042, O R=4.20,95 \% C l: 1.054-16.666)$. Univariate analysis showed statistical significance between the hepatobiliary phase signal group and the FGFR4 mRNA expression, while there was no statistical difference in other qualitative and quantitative features (Table. 4). Further binary logistic regression analysis suggested that signal change in the hepatobiliary phase was an independent indicator for the FGFR4 mRNA expression $(P=0.006)$, and the positive FGFR4 
expression was higher in the heterogeneous signal group than in the hypointensity group $(P \quad=0.008, O R=2.646,95 \% \mathrm{Cl}: 1.291-5.241)($ Tables $5 \& 6)$.

\section{Discussion}

FGFR4 is the target of the first-line targeted drug Lenvatinib in targeted cancer therapy, and can be used as an independent predictor of the therapeutic response to Lenvatinib[9]. To date, the relationship between the FGFR4 gene expression in tumor tissues and MRI features has not been explored by immunohistochemistry and PCR. This study analyzed the preoperative Gd-EOB-DTPA-enhanced MRI features and assessed their correlation with FGFR4 gene expression level in patients with HCC. The major novel findings were summarized as follows: (1) There was significant correlation between the FGFR4 gene expression and Gd-EOB-DTPA-enhanced MRI features in HCC; (2) Heterogeneous signal intensity in the hepatobiliary phase on enhanced MRI was independent indicator for high FGFR4 expression in HCC; (3) The presence of Intratumoral vessels in the arterial phase on enhanced MRI were independent indicators for high FGFR4 protein expression in HCC; (4) These findings suggested the potential for Gd-EOB-DTPA-enhanced MRI as noninvasive procedure in an evaluation of FGFR4 in patients with HCC. 
With increasing evidence that differences in gene expression can influence responses to Lenvatinib treatment, it may be interesting to predict the response rate. Notably, we found that the signs in the hepatobiliary phase and the presence of intratumoral blood vessels were independent predictors of the FGFR4 gene expression. The expression levels of FGFR4 were higher in the HCC patients with heterogeneous signal in the hepatobiliary phase and infantile vessels in the arterial phase compared with those patients with hypointensity in the hepatobiliary phase and no infantile vessels in the arterial phase.

Extensive previous studies have demonstrated that FGFR4 plays a key role in multiple cellular processes, and is involved in the development and progression of various cancers[15], such as malignant melanoma[16], breast cancer[17] and renal cell carcinoma[18], as well as $\mathrm{HCC}[19]$, making it an attractive target in the development of novel and effective anticancer therapies. Additional research work has been applied to the development of selective inhibitors targeting FGFR4, and the findings showed particular promise as monotherapy or adjuvant therapy against cancer. Therefore, HCC with high FGFR4 expression can promote angiogenesis and facilitate rapid tumor proliferation and growth which is consistent with the results in this study that the expression of FGFR4 in 
HCC with intratumoral vessels is higher than that in HCC without intratumoral vessels. This may be due to the role of FGFR4 gene in mediating tumor angiogenesis. The performance of contrast agent gadoxetic acid in hepatocarcinoma tissues during enhanced MRI is mainly related to the uptake and excretion of gadolinium acid by proteins such as organic anion transport polypeptide (OATP), multi-drug resistance associated protein 2(MRP2) and MRP3, resulting in the corresponding changes in the signal intensity in the hepatobiliary phase[21]. FGFR4 for tissue proliferation repair, may lead to cancer the inconsistency of the ingredients in the organization, and to the corresponding organization contrast agent to absorb and discharge distribution change of transporter, which may cause enhancement scanning courage period mixed high and low signal. In our study, we found that the decrease percentage of T1 (T1D\%) was not significantly correlated with the expression of FGFR4. There are possibilities that the small sample size was relative small, or the relatively small number of T1mapping tests conducted in this study.

Lenvatinib and some other anticancer drugs specifically targeting FGFR4 prevent angiogenesis and inhibit tumor growth through downstream MAP kinase and Akt signaling pathways[19]. Therefore, the use of Lenvatinib or specific FGFR4 inhibitors may have better therapeutic effect in patients with high expression of FGFR4 gene. Previous studies on the correlation 
between the expression of this molecule and imaging were limited and the findings were inconsistent in previous studies [22-25]. Our study, together with the above previous findings of others, suggested that HCC patients with MRI features reflecting high FGFR4 expression, including heterogeneous signs in the hepatobiliary phase and the presence of Intratumoral vessels in the arterial phase on enhanced MRI, can be expected to have better therapeutic effects with corresponding drugs. Therefore, this study has clinical significance for the selection of appropriate molecular therapeutic drugs for liver cancer patients in clinical practice.

This study may have some limitations. Firstly, there may be potential selection bias in pathological sampling due to the nature of retrospective study. Secondly, the study population of MRI quantitative characteristics is relatively small, and not all patients can be evaluated by T1 mapping. Further studies on larger multicenter cohorts are needed. Thirdly, the results of our current study are not completely consistent with the results of immunohistochemistry and RT-PCR, resulting in inconsistency with the correlation analysis results of the corresponding MRI features which requires an equal sample size or multi-center cooperative analysis in the future study.

\section{Conclusion}


Taken together, the findings of this study have indicated that signal intensity heterogeneous in the hepatobiliary phase and the presence of Intratumoral vessels in the arterial phase on enhanced MRI reflect the high FGFR4 expression in HCC. Considering FGFR4 as an independent predictor of targeted drug therapy such as Lenvatinib as well as a variety of small molecule drug targets, Gd-EOB-DTPA-enhanced MRI is potential modality in treatment selection and efficacy evaluation of targeted therapies for HCC patients. Further researches are needed to validate the findings in the future studies, and eventually to improve our care for patients with HCC.

\section{Abbreviations:}

$\triangle \mathrm{Ct}$ ca: $\triangle \mathrm{Ct}$ value of carcinoma; caliv: $\triangle \mathrm{Ct}$ value of liver tissue adjacent to carcinoma; GADPH: glyceraldehyde-3-phosphate dehydrogenase

\section{Ethics approval and consent to participate}

The research was approved by the Ethic Committee of the First Affiliated Hospital of Guangxi Medical University (Guangxi, China), an equivalent to the 
Institutional Review Board (IRB). This study was conducted in accordance with ethical guidelines for human research and in accordance with the Health Insurance Portability and Accountability Act (HIPAA). This study confirms that informed consent has been obtained from all subjects or, if subjects are under 18 years of age, from parents and/or legal guardians.

\section{Consent for publication}

Not applicable.

\section{Competing interests}

The authors (Zhiqing Mo, Liling Long, Hao Ding, Xiaojiao Zhou) declare that they have no competing interests.

\section{Funding}

This study was supported by the National Natural Science Foundation of China (No. 82060310).

\section{Authors' contributions}

The First author: Zhiqingmo, who was responsible for the experimental process, specimen collection and manuscript writing. The corresponding author: Liling Long,who was responsible for reviewing articles and supervising the experiment process. The second author: Hao Ding, responsible for chart processing.The third author : Xiaojiao Zhou, 
provided experimental guidance and participated in the specimen collection.

\section{Data Availability}

The data supporting the conclusions of this study are available from the corresponding author upon a reasonable request.

\section{Conflicts of Interests}

The authors declare no competing interests.

\section{Acknowledgement}

Not Applicable.

\section{Authors' information}

All authors are from the same work unit: Department of Radiology, the First Affiliated Hospital of Guangxi Medical University, Nanning 530021, China

\section{References}

1. Dasgupta P, Henshaw C, Youlden DR, Clark PJ, Aitken JF, Baade PD. Global Trends in Incidence Rates of Primary Adult Liver Cancers: A Systematic Review and Meta-Analysis. Front Onco/2020, 10:171.

2. Cervello M, Emma MR, Augello G, Cusimano A, Giannitrapani L, Soresi M et al. New landscapes and horizons in hepatocellular carcinoma therapy. Aging (Albany NY) 2020, 12(3):3053-3094.

3. Lu X, Chen H, Patterson AV, Smaill JB, Ding K. Fibroblast Growth Factor Receptor 4 (FGFR4) Selective Inhibitors as Hepatocellular Carcinoma Therapy: Advances and 
Prospects. J Med Chem 2019, 62(6):2905-2915.

4. Ho HK, Pok S, Streit S, Ruhe JE, Hart S, Lim KS et al. Fibroblast growth factor receptor 4 regulates proliferation, anti-apoptosis and alpha-fetoprotein secretion during hepatocellular carcinoma progression and represents a potential target for therapeutic intervention. J Hepato/ 2009, 50(1):118-127.

5. Heinzle C, Erdem Z, Paur J, Grasl-Kraupp B, Holzmann K, Grusch M et al. Is fibroblast growth factor receptor 4 a suitable target of cancer therapy? Curr Pharm Des 2014, 20(17):2881-2898.

6. Personeni N, Pressiani T, Rimassa L. Lenvatinib for the treatment of unresectable hepatocellular carcinoma: evidence to date. J Hepatocell Carcinoma 2019, 6:31-39.

7. Kobayashi M, Kudo M, Izumi N, Kaneko S, Azuma M, Copher R et al. Cost-effectiveness analysis of lenvatinib treatment for patients with unresectable hepatocellular carcinoma (uHCC) compared with sorafenib in Japan. J Gastroentero/2019, 54(6):558-570.

8. Burrell RA, McGranahan N, Bartek J, Swanton C. The causes and consequences of genetic heterogeneity in cancer evolution. Nature 2013, 501(7467):338-345.

9. Yamauchi M, Ono A, Ishikawa A, Kodama K, Uchikawa S, Hatooka H et al. Tumor Fibroblast Growth Factor Receptor 4 Level Predicts the Efficacy of Lenvatinib in Patients With Advanced Hepatocellular Carcinoma. Clin Trans/ Gastroentero/ 2020, 11(5):e00179.

10. Gillies RJ, Kinahan PE, Hricak H. Radiomics: Images Are More than Pictures, They Are Data. Radiology 2016, 278(2):563-577.

11. Dong Z, Huang K, Liao B, Cai H, Dong Y, Huang M et al. Prediction of sorafenib treatment-related gene expression for hepatocellular carcinoma: preoperative MRI and histopathological correlation. Eur Radio/ 2019, 29(5):2272-2282.

12. Hectors SJ, Wagner M, Bane O, Besa C, Lewis S, Remark R et al. Quantification of hepatocellular carcinoma heterogeneity with multiparametric magnetic resonance imaging. Sci Rep 2017, 7(1):2452.

13. Hectors SJ, Lewis S, Besa C, King MJ, Said D, Putra J et al. MRI radiomics features predict immuno-oncological characteristics of hepatocellular carcinoma. Eur Radio/2020, 30(7):3759-3769.

14. Zhou X, Long L, Mo Z, Li Y. OATP1B3 Expression in Hepatocellular Carcinoma Correlates with Intralesional Gd-EOB-DTPA Uptake and Signal Intensity on Gd-EOB-DTPA-Enhanced MRI. Cancer Manag Res 2021, 13:1169-1177.

15. Eswarakumar VP, Lax I, Schlessinger J. Cellular signaling by fibroblast growth factor receptors. Cytokine Growth Factor Rev 2005, 16(2):139-149.

16. Streit S, Mestel DS, Schmidt M, Ullrich A, Berking C. FGFR4 Arg388 allele correlates with tumour thickness and FGFR4 protein expression with survival of melanoma patients. $\mathrm{Br} \mathrm{J}$ Cancer 2006, 94(12):1879-1886.

17. Roidl A, Berger HJ, Kumar S, Bange J, Knyazev P, Ullrich A. Resistance to chemotherapy is associated with fibroblast growth factor receptor 4 up-regulation. Clin Cancer Res 2009, 15(6):2058-2066.

18. Takahashi A, Sasaki H, Kim SJ, Kakizoe T, Miyao N, Sugimura T et al. Identification of receptor genes in renal cell carcinoma associated with angiogenesis by differential hybridization technique. Biochem Biophys Res Commun 1999, 257(3):855-859.

19. Raja A, Park I, Haq F, Ahn SM. FGF19-FGFR4 Signaling in Hepatocellular Carcinoma. Cel/s 
2019, 8(6):536.

20. Lang L, Teng Y. Fibroblast Growth Factor Receptor 4 Targeting in Cancer: New Insights into Mechanisms and Therapeutic Strategies. Cel/s 2019, 8(1):31.

21. Poetter-Lang S, Bastati N, Messner A, Kristic A, Herold A, Hodge JC et al. Quantification of liver function using gadoxetic acid-enhanced MRI. Abdom Radiol (NY) 2020,

45(11):3532-3544.

22. Kong D, Liu C, Miao X, Wang Y, Ding X, Gong W. Current statuses of molecular targeted and immune checkpoint therapies in hepatocellular carcinoma. Am J Cancer Res 2020, 10(5):1522-1533.

23. Feun LG, Li YY, Wu C, Wangpaichitr M, Jones PD, Richman SP et al. Phase 2 study of pembrolizumab and circulating biomarkers to predict anticancer response in advanced, unresectable hepatocellular carcinoma. Cancer 2019, 125(20):3603-3614.

24. McEvoy SH, McCarthy CJ, Lavelle LP, Moran DE, Cantwell CP, Skehan SJ et al. Hepatocellular carcinoma: illustrated guide to systematic radiologic diagnosis and staging according to guidelines of the American Association for the Study of Liver Diseases. Radiographics 2013, 33(6):1653-1668.

25. Xia W, Chen Y, Zhang R, Yan Z, Zhou X, Zhang B et al. Radiogenomics of hepatocellular carcinoma: multiregion analysis-based identification of prognostic imaging biomarkers by integrating gene data-a preliminary study. Phys Med Bio/ 2018, 63(3):035044. 


\section{Figure legends}

Figure 1 Gd-EOB-DTPA enhanced MRI features in HCC patients. (A) Hypo-intensity in the hepatobiliary phase; (B) Heterogeneous signal intensity of both hypo- and hyper-intensity in the hepatobiliary phase; (C) Intratumoral hemorrhage; (D) Intratumoral vessel; (E-F) Intratumoral fat (white arrow) on the in-phase and out-of-phase images.

Figure 2 Immunohistochemical staining of FGFR4 in HCC tissues. Immunohistochemical staining for FGFR4 was conducted in 59 HCC tissues. Yellow/brown staining was observed on the nucleus, membrane, and cytoplasm. Positive cells were denoted by the black arrow. (A) Immunohistochemical staining of FGFR4 protein expression in histologically normal liver tissues adjacent to HCC; (B) Representative HCC tissues with FGFR4 protein expression $<10 \%$; (C) Representative HCC tissues with FGFR4 protein expression 10\%-50\%; (D) Representative HCC tissues with FGFR4 protein expression $\geq 50 \%$. 
Figure 3 FGFR4 gene expression levels between subgroups in HCC. (A) FGFR4 gene expression levels between two groups with or without Intratumoral vessels in the arterial phase. The protein expression level of FGFR4 was statistically different between the two groups $(P=0.041)$; (B) FGFR4 mRNA expression levels between the hypointense group and the heterogeneous group in the hepatobiliary phase. The FGFR4 mRNA expression was statistically different between the two groups $\left({ }^{*} \mathrm{P}=0.005\right)$.

\section{Figures}

\section{Figure 1}

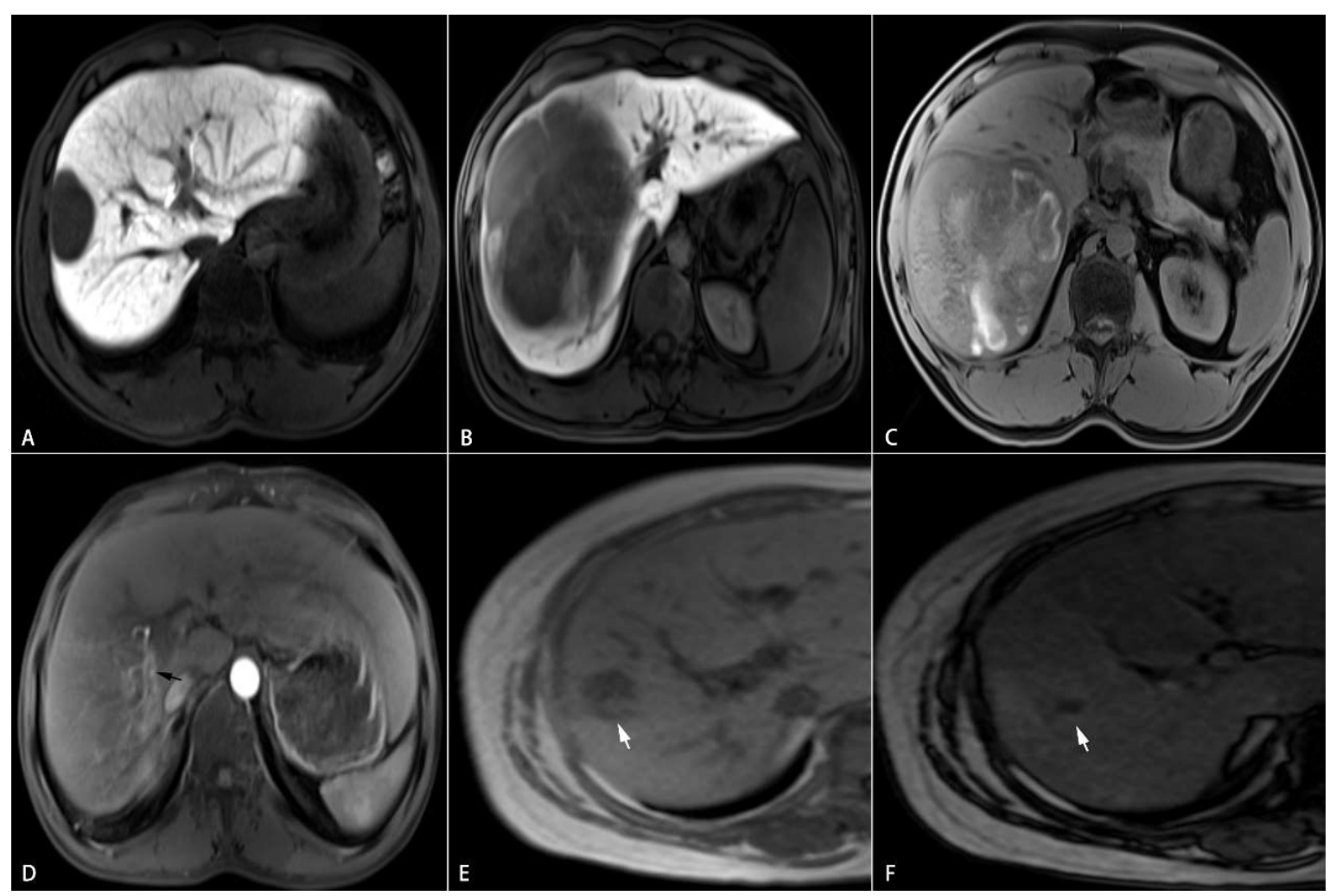




\section{Figure 2}
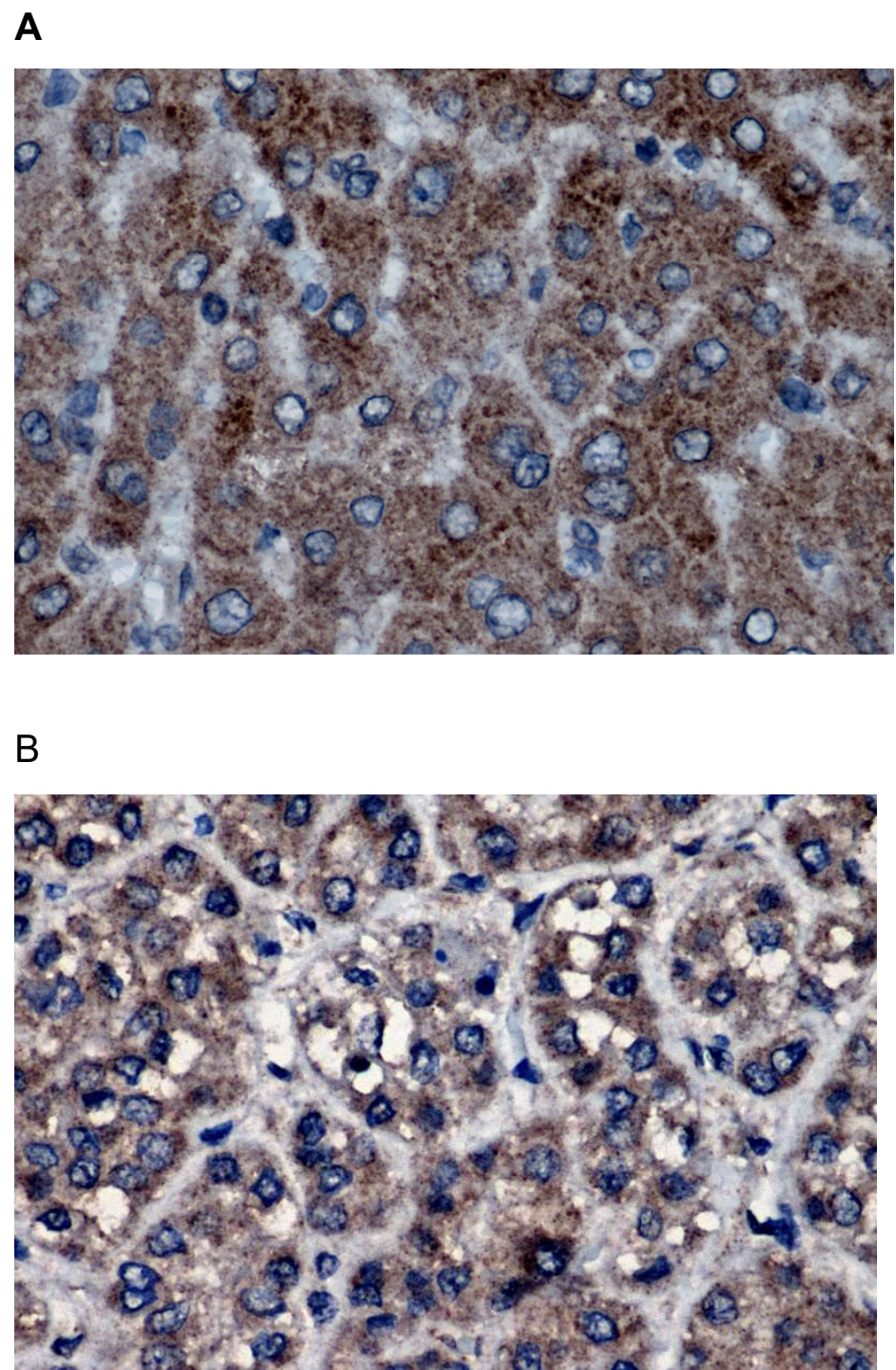

C 


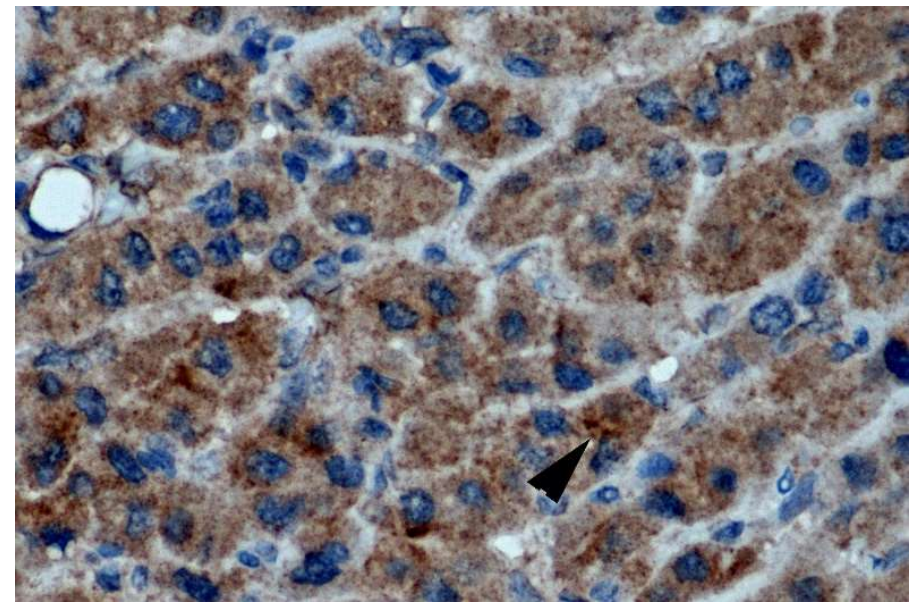

D

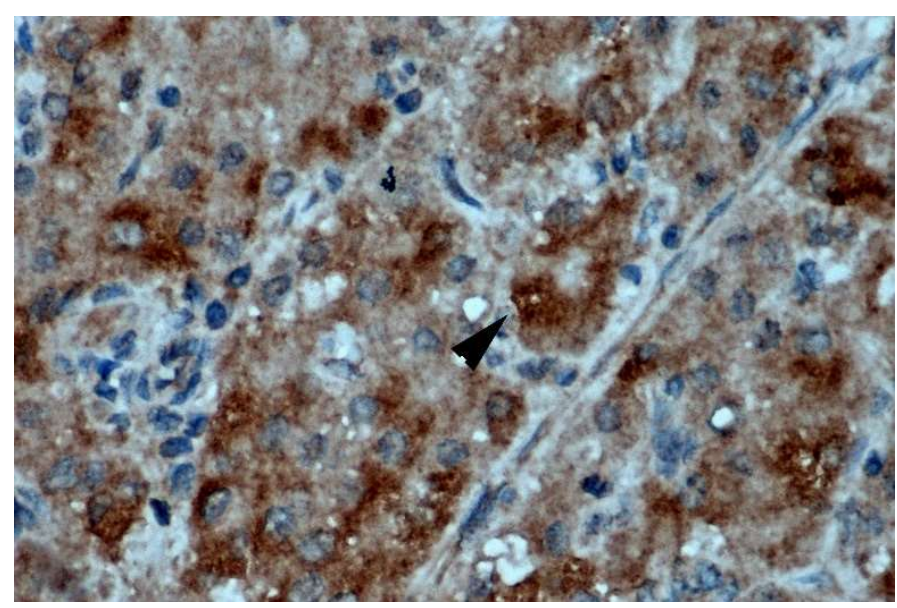




\section{Figure 3}

(A)

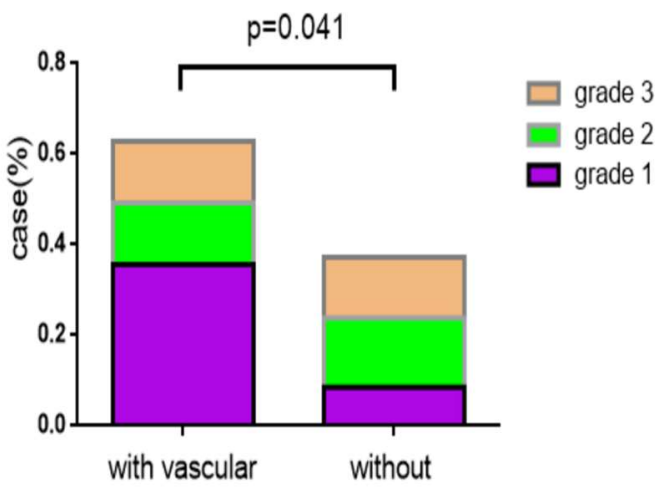

Intratumoral vascular and FGFR4 expression of HCC with immunohistochemical

(B) 


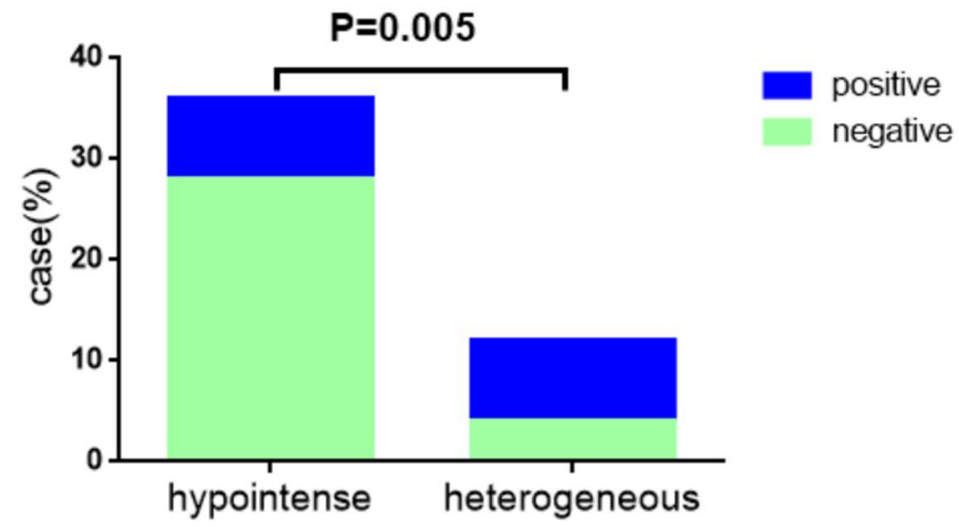




\section{Tables}

Table 1 Qualitative Gd-EOB-DTPA enhanced MRI features in HCC patients

\begin{tabular}{|c|c|c|}
\hline qualitative MRI features & $\begin{array}{l}\text { MRI features } \\
\text { (59 cases) }\end{array}$ & Number \\
\hline & Tumor thrombus & \\
\hline & Yes & 4 \\
\hline & No & 55 \\
\hline & T2WI Signal intensity & \\
\hline & homogeneous & 4 \\
\hline & heterogeneous & 55 \\
\hline & hepatobiliary phase & \\
\hline & Signal & \\
\hline & hypointense & 43 \\
\hline & heterogeneous & 16 \\
\hline & Tumor capsule & \\
\hline & Complete & 23 \\
\hline & Incomplete & 13 \\
\hline & None & 18 \\
\hline & Tumor margins & \\
\hline & Smooth solitary nodule & 23 \\
\hline & Protruding nodule & 24 \\
\hline & Fusion of multiple & 11 \\
\hline & infiltrative & 1 \\
\hline & Intratumoral vessels & \\
\hline & Yes & 22 \\
\hline & No & 37 \\
\hline & Intratumoral & \\
\hline & hemorrhage & \\
\hline & Yes & 23 \\
\hline & No & 36 \\
\hline & Intratumoral fat & \\
\hline & Yes & 5 \\
\hline & No & 54 \\
\hline
\end{tabular}


Table 2 Comparison between qualitative Gd-EOB-DTPA enhanced MRI features and FGFR4 protein expression in HCC

\begin{tabular}{|c|c|c|}
\hline \multirow[t]{2}{*}{ MRI features (59 cases) } & \multicolumn{2}{|c|}{ FGFR4 } \\
\hline & $\chi 2$ & $\mathrm{p}$ value \\
\hline \multicolumn{3}{|l|}{ Tumor thrombus } \\
\hline Ye s $n=(10)$ & 0.321 & 0.852 \\
\hline \multicolumn{3}{|l|}{ No $n=(49)$} \\
\hline T2WI Signal intensity & 5.13 & 0.77 \\
\hline \multicolumn{3}{|l|}{ homogeneous $n=(4)$} \\
\hline \multicolumn{3}{|l|}{ heterogeneous $n=(55)$} \\
\hline \multicolumn{3}{|l|}{ hypointense $n=(43)$} \\
\hline \multicolumn{3}{|l|}{ heterogeneous $n=(16)$} \\
\hline Tumor capsule & 1.3 & 0.522 \\
\hline \multicolumn{3}{|l|}{ Complete $n=(23)$} \\
\hline \multicolumn{3}{|l|}{ Incomplete $\mathrm{n}=(13)$} \\
\hline \multicolumn{3}{|l|}{ None $n=(18)$} \\
\hline Tumor margins & 0.11 & 0.946 \\
\hline \multicolumn{3}{|c|}{ Smooth solitary nodule $\mathrm{n}=(23)$} \\
\hline \multicolumn{3}{|l|}{ Protruding nodule $n=(24)$} \\
\hline \multicolumn{3}{|c|}{ Fusion of multiple nodules $n=(11)$} \\
\hline \multicolumn{3}{|l|}{ infiltrative $n=(1)$} \\
\hline Intratumoral vascular & 6.402 & 0.041 \\
\hline \multicolumn{3}{|l|}{ Yes $n=(22)$} \\
\hline \multicolumn{3}{|l|}{ No $n=(37)$} \\
\hline Intratumoral hemorrhage & 1.103 & 0.576 \\
\hline \multicolumn{3}{|l|}{ Yes $n=(23)$} \\
\hline \multicolumn{3}{|l|}{ No $n=(36)$} \\
\hline Intratumoral fat & 0.554 & 0.758 \\
\hline \multicolumn{3}{|l|}{ Yes $n=(5)$} \\
\hline No $n=(54)$ & & \\
\hline
\end{tabular}


Table 3 Correlation between quantitative Gd-EOB-DTPA enhanced MRI features and FGFR4 protein expression in HCC

\begin{tabular}{lll}
\hline MRI features & FGFR4 & \\
\cline { 2 - 3 } & Correlation & p value \\
\hline & coefficient & \\
Tumor size in diameter $(\mathrm{mm})(\mathrm{n}=59)$ & 0.049 & 0.712 \\
Apparent diffusion coefficient [ADC, $(\times$ & 0.079 & 0.553 \\
$\left.\left.10-3 \mathrm{~mm}^{2} / \mathrm{s}\right)\right](\mathrm{n}=59)$ & 0.229 & 0.306 \\
\hline $1 \mathrm{D} \%(\mathrm{n}=22)$ & & \\
\hline
\end{tabular}

Table 4 Logistic regression analysis of Gd-EOB-DTPA enhanced MRI features associated with FGFR4 protein levels in HCC

\begin{tabular}{|c|c|c|c|c|c|c|c|}
\hline \multirow{2}{*}{\multicolumn{2}{|c|}{ Variables }} & \multicolumn{4}{|c|}{$\begin{array}{l}\text { FGFR4 } \\
\text { (grade2) }\end{array}$} & \multicolumn{2}{|c|}{ FGFR4（grade3） } \\
\hline & & $p$ value & OR & $95 \% \mathrm{Cl}$ & $\begin{array}{l}p \\
\text { value }\end{array}$ & OR & $95 \% \mathrm{Cl}$ \\
\hline Intratumoral vessels & & 0.034 & & & & & \\
\hline Intratumoral vessels & (Yes/No) & 0.026 & $\begin{array}{l}4.7 \\
1\end{array}$ & $\begin{array}{l}1.209 \\
18.518\end{array}$ & 0.042 & 4.2 & $1.054,16.666$ \\
\hline
\end{tabular}


Table 5 Comparison between qualitative Gd-EOB-DTPA enhanced MRI features and FGFR4 mRNA expression levels in HCC

\begin{tabular}{lcc}
\hline MRI features (48 cases) & FGFR4 & \\
\cline { 2 - 3 } & $\chi 2$ & p value \\
\hline Tumor thrombus & & \\
Yes $\mathrm{n}=(8)$ & 0.328 & 0.567 \\
No $\mathrm{n}=(40)$ & & \\
T2WI Signal intensity & 0.534 & 0.465 \\
homogeneous $\mathrm{n}=(4)$ & & \\
heterogeneous $\mathrm{n}=(44)$ & & \\
hepatobiliary phase Signal & 7.833 & \\
$\quad$ hypointense $\mathrm{n}=(36)$ & & \\
heterogeneous $\mathrm{n}=(12)$ & & \\
Tumor capsule & 0.005 \\
Complete $\mathrm{n}=(23)$ & & \\
Incomplete $\mathrm{n}=(12)$ & & \\
None $\mathrm{n}=(13)$ & & \\
Tumor margins & 0.674 \\
Smooth solitary nodule $\mathrm{n}=(17)$ & & \\
Protruding nodule $\mathrm{n}=(20)$ & & \\
Fusion of multiple nodules $\mathrm{n}=(10)$ & & \\
infiltrative $\mathrm{n}=(1)$ & & \\
Intratumoral vascular & & \\
Yes $\mathrm{n}=(20)$ & & \\
No $\mathrm{n}=(28)$ & & \\
Intratumoral hemorrhage & & \\
Yes $\mathrm{n}=(19)$ & & \\
No $\mathrm{n}=(29)$ & & \\
Intratumoral fat & & \\
Yes $\mathrm{n}=(5)$ & & \\
No $\mathrm{n}=(43)$ & & \\
\hline
\end{tabular}


Table 6 Logistic regression analysis of Gd-EOB-DTPA enhanced MRI features associated with FGFR4 expression levels in HCC

\begin{tabular}{lrrr}
\hline Variables & p value & OR & $95 \% \mathrm{Cl}$ \\
\hline Hepatobiliary phase Signal & 0.006 & & \\
Heterogeneous vs hypointense (16/32) & 0.008 & 7 & $1.668,29.384$ \\
\hline
\end{tabular}




\section{Figures}
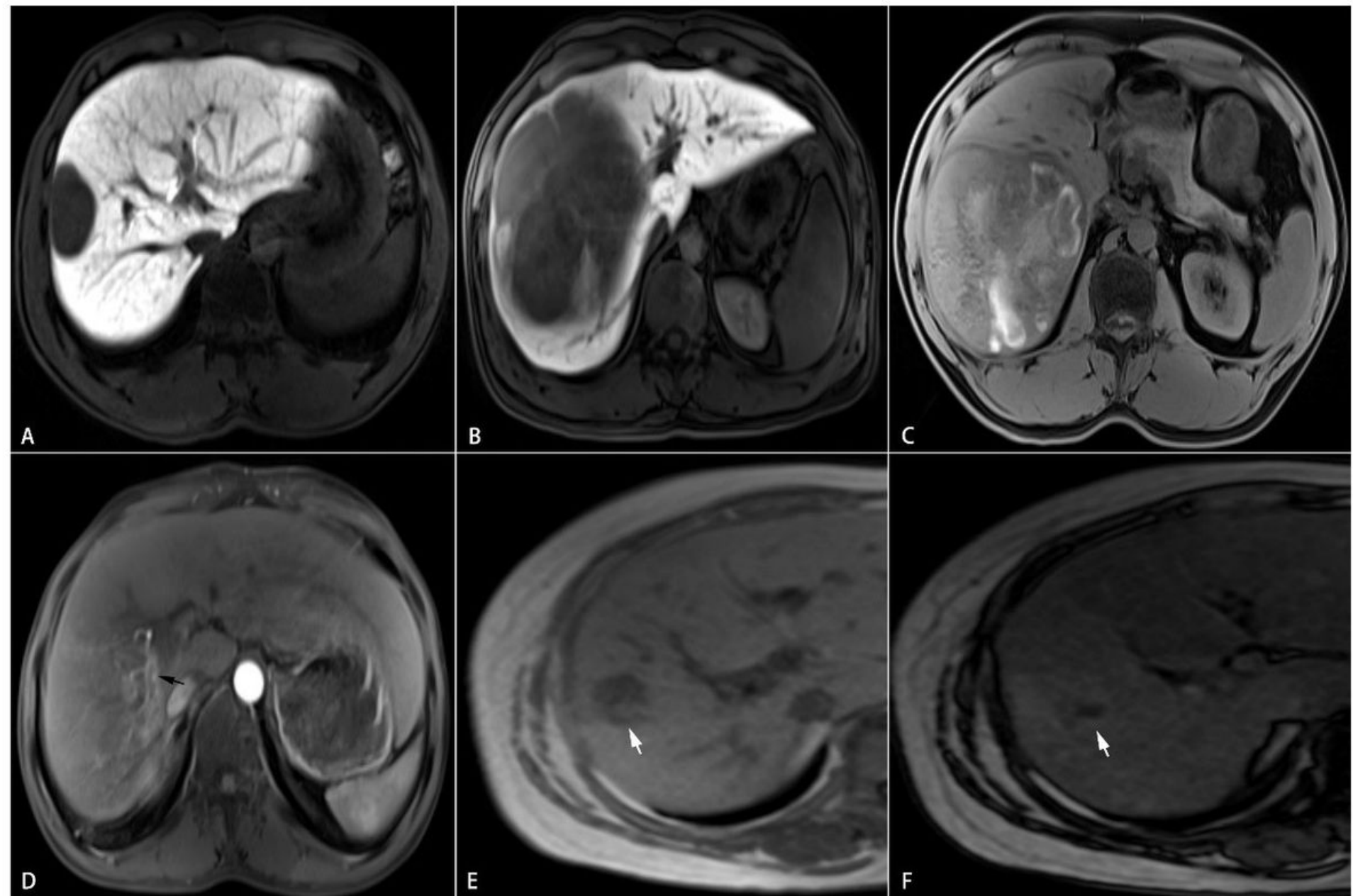

\section{Figure 1}

Gd-EOB-DTPA enhanced MRI features in HCC patients. (A) Hypo-intensity in the hepatobiliary phase; (B) Heterogeneous signal intensity of both hypo- and hyper-intensity in the hepatobiliary phase; (C) Intratumoral hemorrhage; (D) Intratumoral vessel; (E-F) Intratumoral fat (white arrow) on the in-phase and out-of-phase images. 


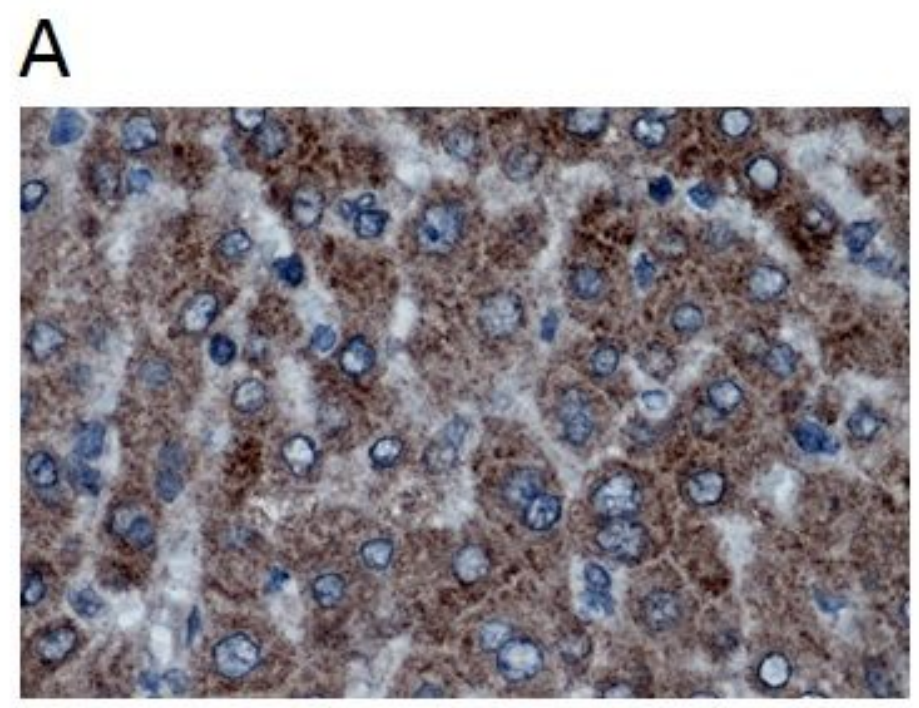

\section{C}

\section{B}

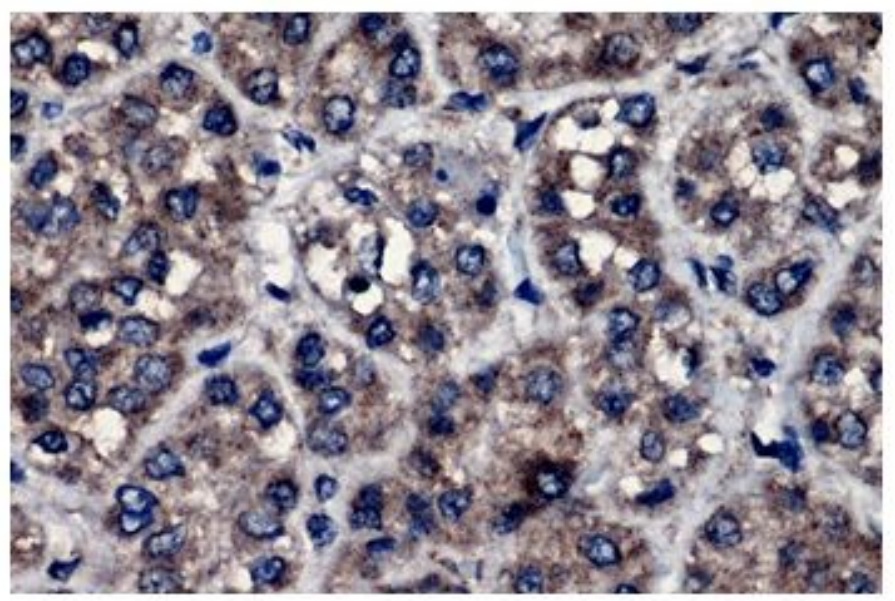

D

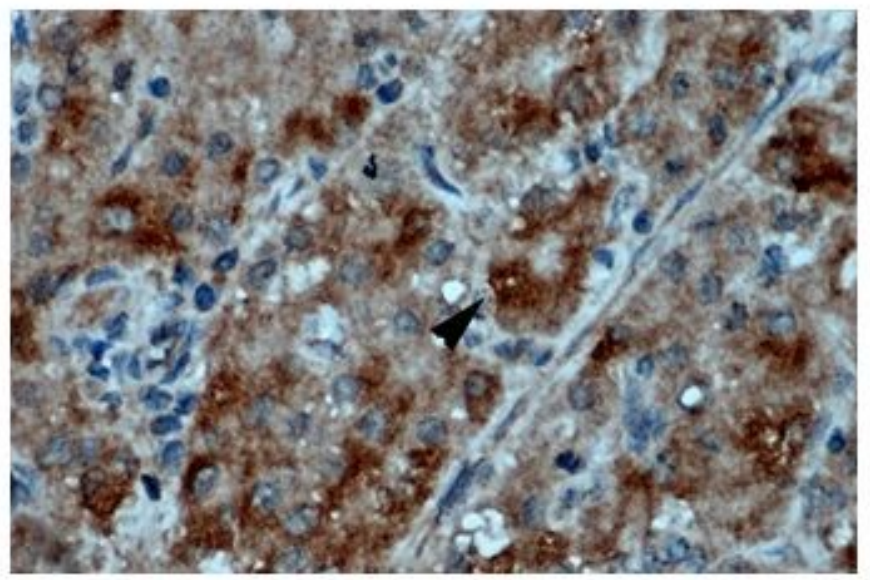

\section{Figure 2}

Immunohistochemical staining of FGFR4 in HCC tissues. Immunohistochemical staining for FGFR4 was conducted in 59 HCC tissues. Yellow/brown staining was observed on the nucleus, membrane, and cytoplasm. Positive cells were denoted by the black arrow. (A) Immunohistochemical staining of FGFR4 protein expression in histologically normal liver tissues adjacent to HCC; (B) Representative HCC tissues with FGFR4 protein expression <10\%; (C) Representative HCC tissues with FGFR4 protein expression 10\%-50\%; (D) Representative HCC tissues with FGFR4 protein expression $\geq 50 \%$. 
(A)

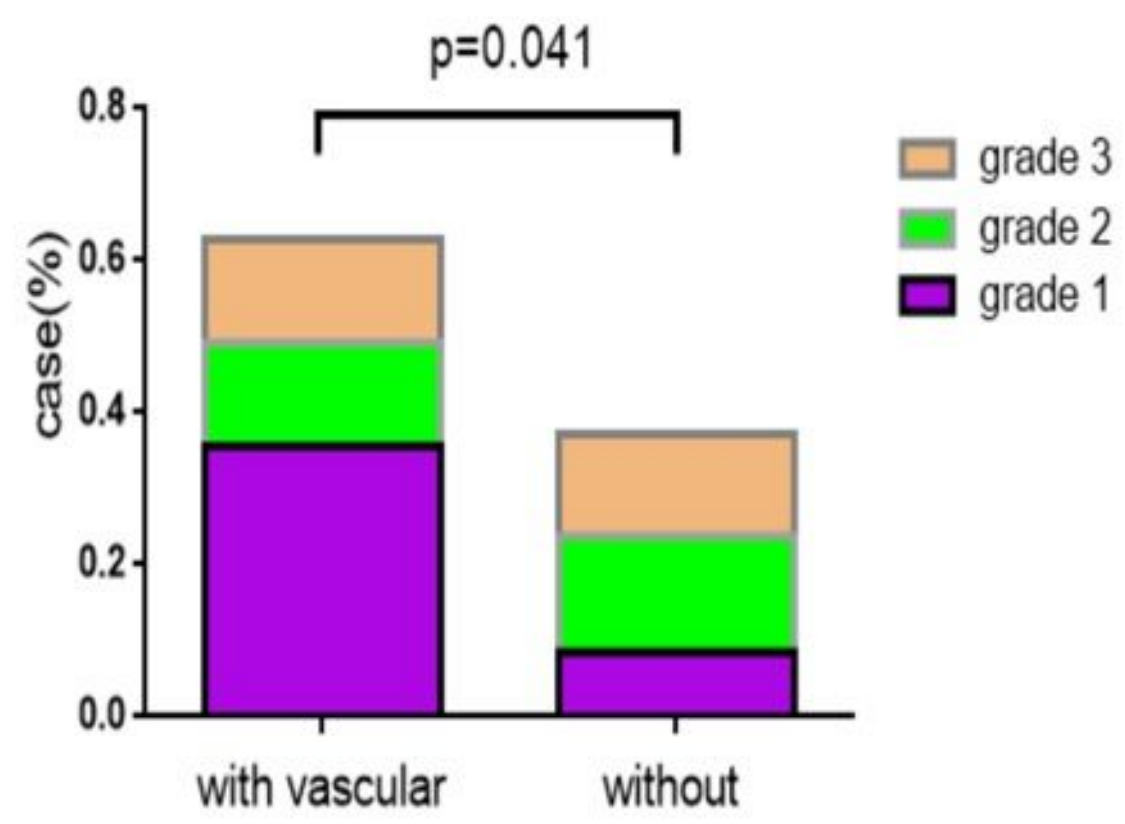

Intratumoral vascular and FGFR4 expression of HCC with immunohistochemical

(B)

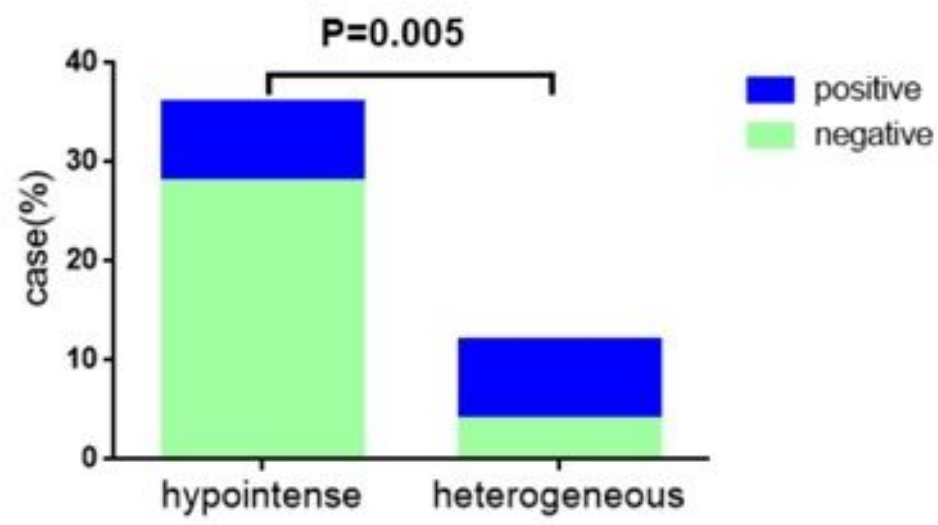

Figure 3

FGFR4 gene expression levels between subgroups in HCC. (A) FGFR4 gene expression levels between two groups with or without Intratumoral vessels in the arterial phase. The protein expression level of FGFR4 was statistically different between the two groups ( $P=0.041)$; (B) FGFR4 mRNA expression levels between the hypointense group and the heterogeneous group in the hepatobiliary phase. The FGFR4 mRNA expression was statistically different between the two groups ( $\left.{ }^{*} \mathrm{P}=0.005\right)$. 